\title{
Femtosecond Photomagnetic Switching of Spins in Ferrimagnetic Garnet Films
}

\author{
Fredrik Hansteen, ${ }^{1,2, *}$ Alexey Kimel, ${ }^{1}$ Andrei Kirilyuk, ${ }^{1}$ and Theo Rasing ${ }^{1}$ \\ ${ }^{1}$ IMM, Radboud University Nijmegen, 6525 ED Nijmegen, The Netherlands \\ ${ }^{2}$ The Norwegian University of Science and Technology, 7491 Trondheim, Norway
}

(Received 10 March 2005; published 21 July 2005)

\begin{abstract}
We demonstrate that intense laser pulses can be used to directly control the spins in ferrimagnetic garnet films. Through an ultrafast and nonthermal photomagnetic effect the magnetocrystalline anisotropy is modified to create a new long-lived equilibrium orientation for the magnetization. Simultaneously, the magnetization is rotated into this new state by precession in a strong transient optically generated magnetic field. All take place within the $100 \mathrm{fs}$ duration of a single laser pulse, thus demonstrating the feasibility of photomagnetic switching on the femtosecond time scale.
\end{abstract}

DOI: 10.1103/PhysRevLett.95.047402

Laser-induced switching and manipulation of the spins in magnetic materials is of great interest for the further development of magnetic storage technology, spin electronics, and quantum computing [1]. It circumvents the need for complex devices for the generation of ultrafast magnetic field pulses [2,3], and could bring the switching speed into the femtosecond regime. The mechanism that couples spins with light, the spin-orbit interaction, is also responsible for the magnetocrystalline anisotropy. Therefore, in materials where this interaction is strong, spins can be controlled with light $[4,5]$ as well as with the crystal field.

Coherent precession is the fastest known way of altering the direction of the macroscopic magnetization $\mathbf{M}$ in a material. An equation of motion describing the process was given by Landau and Lifshitz [6]:

$$
\frac{d \mathbf{M}}{d t}=-\gamma\left(\mathbf{M} \times \mathbf{H}_{\mathrm{eff}}\right) \text {. }
$$

Here $\gamma=17.6 \mathrm{MHz} / \mathrm{Oe}$ is the gyroscopic ratio, and $\mathbf{H}_{\text {eff }}=\mathbf{H}_{\mathrm{a}}+\mathbf{H}_{\mathrm{dem}}+\mathbf{H}_{\text {ext }}$ is the effective magnetic field composed of anisotropy fields, the demagnetizing field, and the externally applied field. The frequency and amplitude of precession is determined by the magnitude of the effective field and by its direction relative to the magnetization, i.e., $\angle\left(\mathbf{M}, \mathbf{H}_{\text {eff }}\right)$, respectively.

Ultrafast manipulation of the magnetization requires means to control the precessional dynamics. This generally involves starting a precession, stopping it, and keeping the magnetization stable along the desired new orientation. Coherent control and even precessional switching on the time scale of a few hundred picoseconds have been demonstrated by different methods based on the shaping of magnetic field pulses $[3,7,8]$. However, the limitations and complexity of these techniques have stimulated the search for alternative ways to create the equivalent of strong, ultrashort, and localized magnetic field pulses $[9,10]$.

A credible solution, unsurpassed in simplicity and speed, can be found in photomagnetic phenomena. According to Eq. (1), the magnetization $\mathbf{M}$ can be stable $\left(\frac{d \mathbf{M}}{d t}=0\right)$ only along the direction of the effective magnetic
PACS numbers: 78.47.+p, 75.30.Gw, 75.40.Gb, 78.20.Ls

field $\mathbf{H}_{\text {eff }}$. An arbitrary equilibrium for $\mathbf{M}$ can thus be created either by means of an external magnetic field or by modification of the material's anisotropy fields. Previously, laser-induced thermal modulation of these fields has been shown to trigger spin reorientation, precessional dynamics and quantum beating [11-15], but nonthermal optical control of the anisotropy on an ultrafast time scale has remained a challenge until now.

The existence of a nonthermal influence of light on the magnetic anisotropy would enable us to create a new equilibrium state for the magnetization. As is shown below, such a long-lived photomagnetic effect indeed exists and can be generated by both linearly and circularly polarized laser light. By simultaneously utilizing the strong magnetic field created by circularly polarized laser pulses $[4,5,16]$, the precession of the magnetization can be controlled and guided into the new equilibrium state, thus allowing complete coherent control of the spin motion by light.

Using an all-optical pump-and-probe technique, employing $100 \mathrm{fs}$ laser pulses at $\lambda=805 \mathrm{~nm}$, we have demonstrated photomagnetic manipulation of spin dynamics in highly transparent magnetic garnet films with in-plane magnetization, $4 \pi M_{s}=550 \mathrm{G}$, grown on $(001)$ oriented substrates of gadolinium gallium garnet $\left(\mathrm{Gd}_{3} \mathrm{Ga}_{5} \mathrm{O}_{12}\right)$. The films studied in this work have thickness 3-8 $\mu \mathrm{m}$ and composition $\mathrm{Lu}_{3-x-y} \mathrm{Y}_{x} \mathrm{Bi}_{y} \mathrm{Fe}_{5-z} \mathrm{Ga}_{z} \mathrm{O}_{12}$ with small amounts of $\mathrm{Pb}$ impurities due to the flux which they are grown from. All results presented in this Letter are from a $7.5 \mu \mathrm{m}$ thick film with $x=0.65, y=0.66$, and $z=1.15$, but the effects that we describe were seen in the whole series of similar samples. This film has small optical absorption $\left(\alpha \approx 20 \mathrm{~cm}^{-1}\right)$, has a refractive index of about 2.3 , and exhibits a Faraday rotation of $2.5^{\circ}$ at $\lambda=805 \mathrm{~nm}$ [17]. It also has an in-plane fourfold symmetric anisotropy field of about $\mathbf{H}_{\mathrm{a}}=50$ Oe. In our experiment the pumpinduced magnetization dynamics was followed in time by measuring the Faraday rotation of the time-delayed and much weaker $\left(I_{\text {pump }} / I_{\text {probe }}>1000\right)$ probe pulses, which in the present geometry [Fig. 1(a)] reflects the change in the $z$ component of M. Depending on whether the polarization 
(a)

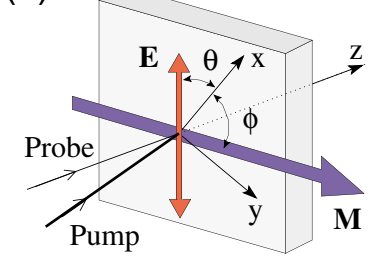

(c)

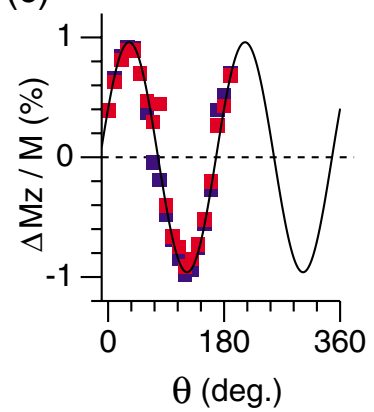

(d)

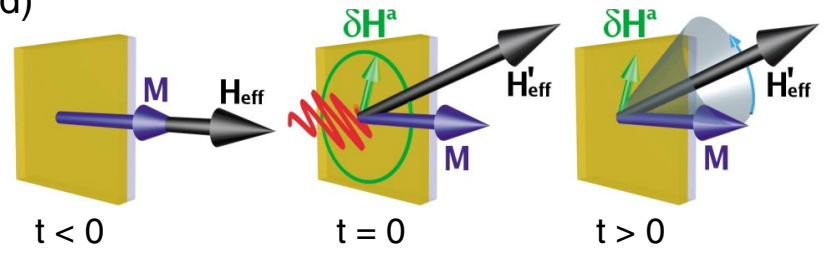

FIG. 1 (color). Coherent precession of the magnetization triggered by a change in anisotropy field $\delta \mathbf{H}^{\mathrm{a}}$ induced by linearly polarized laser pulses. (a) Experimental geometry: A garnet film with in-plane magnetization $\mathbf{M}$ at an angle $\phi$ with respect to the crystallographic $x$ axis. $\theta$ denotes the angle between the plane of polarization of the linearly polarized pump pulses and the $x$ axis. (b) Time dependence of the precession for different planes of polarization $\theta$, with an applied field of $\left|\mathbf{H}_{\text {ext }}\right|=350$ Oe in the plane of the sample. Circles represent measurements and solid lines represent simulations based on the Landau-Lifshitz equation. (c) Precessional amplitude as a function of the plane of polarization. Red and blue symbols represent amplitudes extracted from measurements at $\pm \mathbf{H}_{\text {ext }}$. The solid line is a best fit. (d) An illustration of the excitation process and the subsequent precessional dynamics.

of the pump pulses is linear or circular, two different effects are responsible for triggering the precessional dynamics. Linearly polarized pulses induce a long-lived change of the anisotropy field, the direction of which depends on the orientation of the plane of polarization, while circularly polarized pulses additionally create a strong transient magnetic field along their $k$ vector.

With an external magnetic field applied in the plane of the film, and pumping with linearly polarized $20 \mu \mathrm{J}$ laser pulses, coherent precession of the magnetization was observed. As shown in Fig. 1(b), the amplitude and phase of this optically triggered precession depends on the plane of polarization of the excitation pulse. For every $90^{\circ}$ rotation of the polarization, the precession vanishes and then reap- pears with the opposite phase as the polarization is rotated further. The initial phase of the precession reveals that $\mathbf{M}$ initially moves in the $\pm z$ direction, normal to the film plane. According to Eq. (1), this implies that the photoinduced anisotropy field $\delta \mathbf{H}^{\mathrm{a}}$ is parallel to the film plane.

Figure 1(c) shows the precession amplitude as a function of the pump polarization, $\theta$. The perfectly sinusoidal shape suggests that the magnitude of $\delta \mathbf{H}^{\mathrm{a}}$ is constant and that its direction varies as $2 \theta$, as illustrated by the green circle in Fig. 1(d). When the induced $\delta \mathbf{H}^{\mathrm{a}}$ is along $\mathbf{H}_{\text {eff }}$, then $\frac{d \mathbf{M}}{d t}=$ $-\gamma \mathbf{M} \times\left(\mathbf{H}_{\text {eff }}+\delta \mathbf{H}^{\mathrm{a}}\right)=0$, and no precession is excited as the equilibrium direction is unchanged. The observed polarization dependence rules out the possibility of a simple thermal excitation mechanism. Moreover, the heating induced by the pump pulses is estimated to be well below $5 \mathrm{~K}$ at room temperature.

From the field dependence of the precessional amplitude, and from the sudden and lasting pump-induced decrease in the transmittivity of the sample (not shown here) [18], it is evident that $\delta \mathbf{H}^{\mathrm{a}}$ has a lifetime $\tau$ significantly longer than the time scale of the experiment, i.e., $\tau \gg$ 3 ns.

Photomagnetic effects are known to exist in garnets [1922], but have not previously been observed on this time scale. Changes in magnetocrystalline anisotropy are caused by optically induced electron transfer between ions on nonequivalent sites in the crystal. Effectively, this leads to a redistribution of ions with an accompanying change in the magnetocrystalline anisotropy. Because this is an electronic process, it can take place on the subpicoseond time scale.

Phenomenologically our observations of photoinduced change in anisotropy (Fig. 1) can be described as

$$
\delta H_{i}^{a}(0)=\chi_{i j k l} E_{j}(\omega) E_{k}(\omega) M_{l}(0) .
$$

Here $\chi_{i j k l}$ is a fourth rank polar tensor, which is allowed in media of any symmetry, $E$ is the electric field of the light, and $M$ is the magnetization of the garnet film. When taking the experimental geometry and the symmetry of $\chi_{i j k l}$ for the $4 \mathrm{~mm}$ point group of our samples into account, the following expressions for the optically induced anisotropy field is obtained:

$$
\begin{aligned}
& \delta H_{x}^{a} \propto E^{2} M_{s} \chi[\sin 2 \theta \sin \phi+\cos 2 \theta \cos \phi], \\
& \delta H_{y}^{a} \propto E^{2} M_{s} \chi[\sin 2 \theta \cos \phi-\cos 2 \theta \sin \phi] .
\end{aligned}
$$

$\delta H_{i}^{a}$ is the induced field along the $i$ direction, $i=\{x, y\}$ refers to the crystal axes of the sample, and the angles $\phi$ and $\theta$ are defined in Fig. 1(a). $\chi$ denotes the nonzero tensor component of $\chi_{i j k l}$. When restricting the geometry to inplane magnetization and normal incidence, there is no outof-plane component $\delta H_{z}^{a}$, in accordance with our results.

The coherent precession of $\mathbf{M}$ triggered by circularly polarized laser pulses is shown in Fig. 2. The two helicities $\sigma^{ \pm}$give rise to precession with opposite phase, and again 
the initial motion of $\mathbf{M}$ is normal to the film plane. In this case the excitation is caused by the circularly polarized light setting up a strong axial magnetic field through the inverse Faraday effect $[4,5,16]$ :

$$
\delta H_{i}^{\mathrm{F}}=\chi_{i j k}\left(E_{j} E_{k}^{*}-E_{k} E_{j}^{*}\right) .
$$

The effect is possible due to strong spin-orbit coupling and does not rely on absorption. For paramagnetic materials $\chi_{i j k}$ is closely related to, and can be calculated from, the Verdet constant [23]. In our ferrimagnetic garnet films we find from the measured amplitude of precession $\Lambda$ that an axial magnetic field $\mathbf{H}^{\mathrm{F}} \approx \omega / \gamma \approx \Lambda / \gamma \Delta t$ of about $0.6 \mathrm{~T}$ is created by circularly polarized laser pulses of power density $10^{11} \mathrm{~W} / \mathrm{cm}^{2}$ during their $\Delta t=100 \mathrm{fs}$ presence in the sample. Both this effect and the effect of photoinduced anisotropy were found to depend linearly on the pump intensity [18].

The field $\mathbf{H}^{\mathrm{F}}$ is much stronger than any other magnetic fields involved and, consequently, dominates while present. During the 100 fs presence of this strong out-ofplane magnetic field pulse, the magnetization of the film precesses approximately $0.6^{\circ}$ to a new in-plane orientation. The direction of this precession is determined by the direction of $\mathbf{H}^{\mathrm{F}}$, i.e., by the helicity of the pulse. The phase of the subsequent precessional dynamics is determined by the torque $\mathbf{M} \times \mathbf{H}_{\text {eff }}$, which is oppositely directed for $\sigma^{+}$

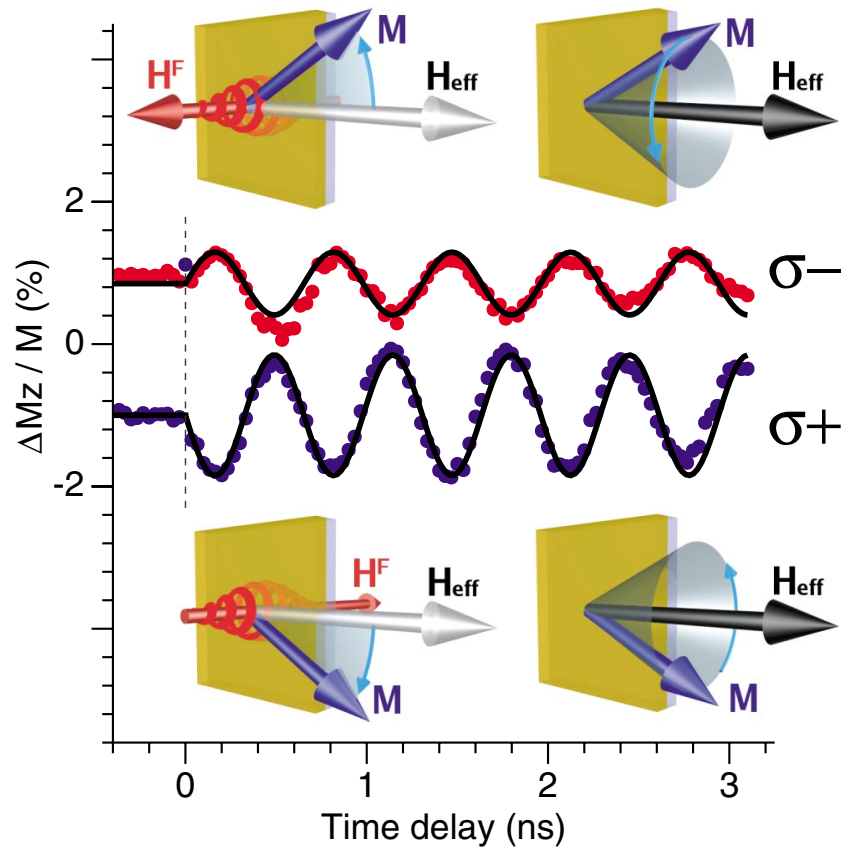

FIG. 2 (color). Precession of the magnetization following excitation with circularly polarized light. The two helicities $\sigma^{+}$ and $\sigma^{-}$give rise to precession with opposite phase and different amplitude. During the presence of the laser pulse $0<t<100 \mathrm{fs}$ the magnetization precesses in the dominating axial field $\mathbf{H}^{\mathrm{F}}$. Subsequent precession takes place in the effective magnetic field composed of anisotropy fields and the externally applied field. and $\sigma^{-}$, and causes $\mathbf{M}$ to initially move in the $+z$ or $-z$ direction, respectively.

It can be noted in Fig. 2 that the precessional amplitude is different for the two helicities. The origin of this asymmetry is found by analyzing Eq. (2) for the situation of excitation by circularly polarized light $\mathbf{E}=\frac{E_{0}}{\sqrt{2}}(\hat{x}+i \hat{y})$, showing that also in this case a contribution $\delta \mathbf{H}^{\mathrm{a}}$ exists. The direction of this anisotropy contribution is determined only by the angle $\phi$ between the magnetization and the $x$ axis, and is independent of the helicity of the incident light. This long-lived photoinduced anisotropy field is created

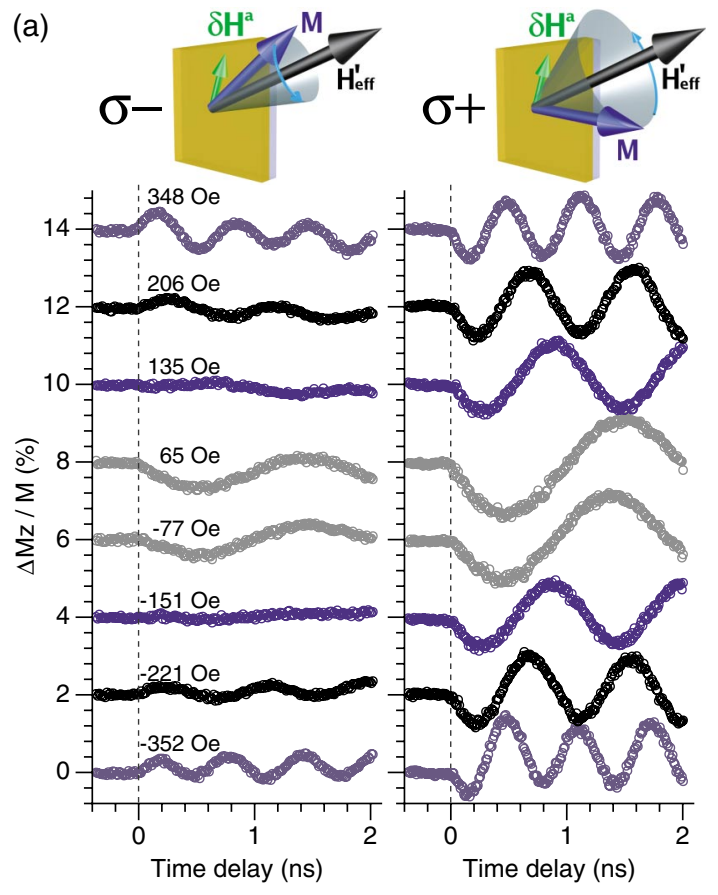

(b)
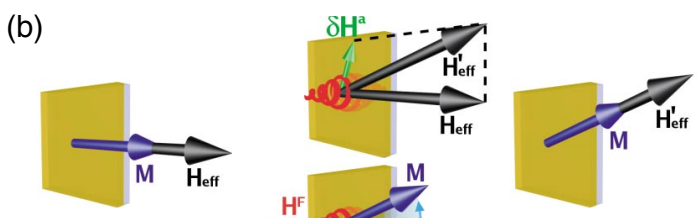

Initial state $\mathrm{t}<0$

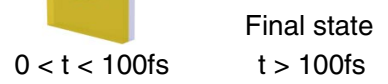

FIG. 3 (color). All-optical switching of the magnetization within 100 fs. (a) Precession of the magnetization triggered by left- and right-hand circularly polarized laser pulses at different values of the in-plane applied magnetic field. For the $\sigma^{-}$helicity, at an applied field of $\sim \pm 150 \mathrm{Oe}$, no precession is observed due to a perfect balance of the two photomagnetic effects. (b) Graphical illustration of the switching process: Initially at $t<0$ the magnetization is along $\mathbf{H}_{\text {eff }}$. During the presence of the laser pulse $0<t<100 \mathrm{fs}$ photoinduced modification of the anisotropy fields leads to a new metastable equilibrium along $\mathbf{H}_{\text {eff }}^{\prime}$. Simultaneously, the strong optically generated field $\mathbf{H}^{\mathrm{F}}$ causes the magnetization to precess into the new state. After $t>$ $100 \mathrm{fs}$ the optical pulse is gone and the $0.6^{\circ}$ switching of $\mathbf{M}$ is complete. 
simultaneously with the transient $\mathbf{H}^{\mathrm{F}}$ field by the same laser pulse. The precession of $\mathbf{M}$ during $0<t<100 \mathrm{fs}$ is thus either towards $\left(\sigma^{-}\right)$or away from $\left(\sigma^{+}\right)$the new effective field $\mathbf{H}_{\text {eff }}^{\prime}=\mathbf{H}_{\text {eff }}+\delta \mathbf{H}^{\mathrm{a}}$, giving rise to a precession with a smaller or larger amplitude in the time $t>$ 100 fs after the pulse; see Fig. 3.

A proper combination of both optically induced fields $\delta \mathbf{H}^{\mathrm{a}}$ and $\mathbf{H}^{\mathrm{F}}$ now allows for complete control and switching of the magnetization vector $\mathbf{M}$. If, after precessing for $100 \mathrm{fs}$ in the strong axial field $\mathbf{H}^{\mathrm{F}}$, we manage to let the magnetization end up exactly along the new equilibrium state $\mathbf{H}_{\text {eff }}^{\prime}=\mathbf{H}_{\text {eff }}+\delta \mathbf{H}^{\mathrm{a}}$ simultaneously created by the optically induced anisotropy field, then no further precession will take place, as $\mathbf{M} \times \mathbf{H}_{\text {eff }}^{\prime}=0$. Because the direction of $\delta \mathbf{H}^{\mathrm{a}}$ depends only on the initial angle $\phi$ of the magnetization with respect to the crystal axes, it can be tuned by rotating the sample with respect to the applied field. Alternatively, since the initial equilibrium of $\mathbf{M}$ is along $\mathbf{H}_{\text {eff }}$, which is determined by the balance between the magnetocrystalline anisotropy field $\mathbf{H}_{\mathrm{a}}$ and the externally applied field, it can also be tuned simply by varying the strength of the applied field.

In Fig. 3(a) the coherent precession of the magnetization following excitation with pulses of helicity $\sigma^{-}$and $\sigma^{+}$is shown for different values of the externally applied magnetic field. The amplitude of precession is consistently larger in the case of $\sigma^{+}$, as during $0<t<100 \mathrm{fs} \mathbf{M}$ precesses away from the new equilibrium created by $\delta \mathbf{H}^{\mathrm{a}}$, as explained above. For pulses of helicity $\sigma^{-}$this precession is towards the new equilibrium, leading to a smaller precessional amplitude in the time after the pulse. With an applied field of $\left|\mathbf{H}_{\mathrm{ext}}\right| \approx 150 \mathrm{Oe}$, no precession is triggered due to a perfect balance of two effects: The inplane precession of the magnetization during the $100 \mathrm{fs}$ magnetic field pulse $\mathbf{H}^{\mathrm{F}}$ brings the magnetization exactly to its new equilibrium orientation created by the optically modified anisotropy field. It remains stable in this orientation for several nanoseconds, until the anisotropy field relaxes back to its original state.

In conclusion, we have shown how a new ultrafast and nonthermal photomagnetic effect of optically induced magnetic anisotropy can be combined with optically generated 100 fs magnetic field pulses of $0.6 \mathrm{~T}$ to completely control the magnetization in garnet films. Though only a small $0.6^{\circ}$ rotation has been demonstrated in the present experiment, the underlying concept of coherently preparing an equilibrium state on a femtosecond time scale, and simultaneously rotating the magnetization into it, may lead to new applications in magnetic recording and quantum computing. Moreover, as increasingly shorter laser pulses are being made [24], direct optical switching of magnetic bits at rates of several $\mathrm{THz}$ and optically induced magnetic field pulses of several Teslas are feasible.

The authors thank L. E. Helseth and T. H. Johansen for providing the samples. This work was supported by The
Norwegian Research Council, The European network DYNAMICS, FOM, and NWO.

*Electronic address: fredrik.hansteen@phys.ntnu.no

[1] Semiconductor Spintronics and Quantum Computation, edited by D. D. Awschalom, D. Loss, and N. Samarth (Springer, Berlin, 2002).

[2] Y. Acremann, M. Buess, C.H. Back, M. Dumm, G. Bayreuther, and D. Pescia, Nature (London) 414, 51 (2001).

[3] T. Gerrits, H. A. M. van den Berg, J. Hohlfeld, L. Bär, and Th. Rasing, Nature (London) 418, 509 (2002).

[4] P. S. Pershan, J.P. van der Ziel, and L. D. Malmstrom, Phys. Rev. 143, 574 (1966).

[5] J.P. van der Ziel, P.S. Pershan, and L.D. Malmstrom, Phys. Rev. Lett. 15, 190 (1965).

[6] L. Landau and E. Lifshitz, Phys. Z. Union 8, 153 (1935).

[7] S. Kaka and S.E. Russek, Appl. Phys. Lett. 80, 2958 (2002).

[8] H. W. Schumacher, C. Chappert, P. Crozat, R. C. Sousa, P. P. Freitas, J. Miltat, J. Fassbender, and B. Hillebrands, Phys. Rev. Lett. 90, 017201 (2003).

[9] Y. Kato, R.C. Myers, A.C. Gossard, and D. D. Awschalom, Nature (London) 427, 50 (2004).

[10] I. Tudosa, C. Stamm, A. B. Kashuba, F. King, H. C. Siegmann, J. Stöhr, G. Ju, B. Lu, and D. Weller, Nature (London) 428, 831 (2004).

[11] G. Ju, A. V. Nurmikko, R. F. C. Farrow, R. F. Marks, M. J. Carey, and B. A. Gurney, Phys. Rev. Lett. 82, 3705 (1999).

[12] M. van Kampen, C. Jozsa, J. T. Kohlhepp, P. LeClair, L. Lagae, W. J. M. de Jonge, and B. Koopmans, Phys. Rev. Lett. 88, 227201 (2002).

[13] G. P. Zhang and W. Hübner, Phys. Rev. Lett. 85, 3025 (2000).

[14] A. V. Kimel, A. Kirilyuk, A. Tsvetkov, R. V. Pisarev, and Th. Rasing, Nature (London) 429, 850 (2004).

[15] N. P. Duong, T. Satoh, and M. Fiebig, Phys. Rev. Lett. 93, 117402 (2004).

[16] A. V. Kimel, A. Kirilyuk, P. A. Usachev, R. V. Pisarev, A. M. Balbashov, and Th. Rasing, Nature (London) (to be published).

[17] F. Hansteen, L. E. Helseth, T. H. Johansen, O. Hunderi, A. Kirilyuk, and T. Rasing, Thin Solid Films 455-456C, 429 (2004).

[18] F. Hansteen, A. V. Kimel, A. Kirilyuk, and Th. Rasing (to be published).

[19] R. W. Teale and D. W. Temple, Phys. Rev. Lett. 19, 904 (1967).

[20] A. B. Chizhik, I. I. Davidenko, A. Maziewski, and A. Stupakiewicz, Phys. Rev. B 57, 14366 (1998).

[21] A. Stupakiewicz, A. Maziewski, I. Davidenko, and V. Zablotskii, Phys. Rev. B 64, 064405 (2001).

[22] V. G. Veselago, R. A. Doroshenko, and S. G. Rudov, Zh. Eksp. Teor. Fiz. 105, 638 (1994) [Sov. Phys. JETP 78, 341 (1994)].

[23] M. Y. A. Raja, D. Allen, and W. Sisk, Appl. Phys. Lett. 67, 2123 (1995).

[24] U. Keller, Nature (London) 424, 831 (2003). 